\title{
An Efficient Routing Algorithm for Opportunistic Networks based on Network Coding
}

\author{
Yukun Yao, Zhihu Liu, Zhi Ren, Yadi Wen \\ Chongqing Key Lab of Mobile Communications Technology, Chongqing University of Posts and Telecommunications, \\ Chongqing, 400065, China \\ E-mail: sunsiant@163.com
}

\begin{abstract}
To address the problems in Opportunistic Networks that nodes employing routing algorithms based on epidemic mechanism can't sense their neighboring nodes timely and many redundant data packets permeate through the network, an efficient routing algorithm based on XOR network coding was proposed (Xor Network Coding based Epidemic Routing, XNCER). When a node gets a Hello message, it broadcasts, instead of unicasting, a SV(Summary Vector)packet, in return, to all its neighboring nodes. Therefore, the data transmission can be initiated immediately, and the XOR network coding can also be leveraged so as to enhance the efficiency of data transmission. Moreover, the packets destined to the one hop neighboring nodes obtain the priority in transmission, and are determined whether to be deleted based on the ACK mechanism running in the MAC layer in order to cut down the memory overhead and restrain redundant packets from dissemination. Theoretical analysis and extensive simulation results show that the new algorithm obtains an overall improvement in terms of network overhead, average end-toend delay as well as delivery rate. The novel algorithm can effectively solve the problems of sensing neighboring nodes and data redundancy.
\end{abstract}

Keywords-opportunistic networks; routing algorithms; network coding; overhead; delay; delivery rate

\section{INTRODUCTION}

Routing algorithms based on epidemic mechanism provide an reliable paradigm for data transmission in opportunistic networks[1][2]. However, the epidemic routing is flooding-like, resulting in badly need for network resource. Moreover, epidemic routing takes advantage of the pairwise contact of two nodes, paying no consideration of local network topology, and can't find the neighboring nodes timely. To data, network coding has been a significant research direction for efficient data transmission in opportunistic networks[3]. Compared with the conventional carry-forward method[4], network coding makes use of the carry-coding-forward paradigm, combining several data flows from different nodes into one data flow, and then forwards the combined data flow, so as to increase the network throughput, shorten the end-to-end delay, and strengthen the network robust.

To find the neighboring nodes timely, cut down the network overhead and shorten the end-to-end delay in epidemic routing, an efficient routing algorithm based on XOR network coding was proposed (Xor Network Coding based Epidemic Routing, XNCER). When a node gets a
Hello message, it sends a SV(Summary Vector)packet, in return, to all its neighboring nodes by means of broadcast. Then, the data transmission can be initiated immediately, and the XOR network coding can also be leveraged when the node has more than one neighboring nodes, enhancing the efficiency of data transmission. Moreover, the packets destined to one hop neighboring nodes of the node obtain the priority in transmission, and are determined whether to be deleted based on the ACK mechanism running in the MAC layer in order to cut down the memory overhead and restrain redundant packets from dissemination. The performance of the novel XNCER algorithm was analyzed and verified as well.

This paper is organized as follows: We review the related work in section 2. Section 3 gives an introduction of basic epidemic routing. Then, section 4 formulates the proposed XNCER algorithm. In the following section 5, simulation and analysis that confirm the validity of the proposed scheme are performed and section 6 will be the conclusion of the whole paper.

\section{RELATED WORK}

To increase the efficiency of data packet transmission, while cutting down the node energy consumption, Wait and Epidemic routing protocol (WE)was put forward[5]. WE separates the data transmission into two stages, that is, wait stage and epidemic stage. The wait stage takes the Direct Delivery routing scheme(DD)[6], while the epidemic stage leverages the constrained epidemic routing. However, the algorithm takes little consideration to the network information resource, leading to large transmission delay.

Katti et al. [7] proposed a localized wireless network coding heuristic called COPE(Completely Opportunity Encoding). In COPE, nodes store overheard or relay data packets and exchange the list of possessed packets with their one hop neighboring nodes via piggy-backing or beaconing. As one node wants to transmit a packet, it greedily mixes as many packets in its output buffer as possible by the XOR operation if each XORed packet is the only one unknown among all XORed packets to the packet's next hop, and then the coded packet is broadcasted. Wang et al. proposed a scheme for data dissemination based on XOR network coding, and can effectively cut down the node energy consumption[8]. The random linear network coding (RLC) has also been used in epidemic routing protocol[9][10][11]. Theoretical analysis and extensive simulation results showed that, the RLC can remarkably shorten the network delay in 
the case of constrained bandwidth and limited node buffer, so as to enhance the reliability of the network. On the contrary, the RLC also increases the transmission delay and the extra resource consumption in the case of sufficient bandwidth and node buffer. That's because that, the RLC increases the computational complexity of the intermediate nodes, and the coded packets received must be adequate enough so as to retrieve the original packets.

\section{Model \& PROBLEM}

\section{A. Network and Routing Models}

Definition 1 Network Model: Mathematical model in opportunistic networks is defined as follows. $G=(V, E)$. Here, the node set $V=\left\{v_{1}, v_{2}, \ldots, v_{n}\right\}, \mathrm{n}$ denotes the number of nodes in the network, and $n>1$; The link set $E=\varnothing \cup\left\{e_{1}, e_{2}, \ldots, e_{m}\right\}$, where, $e_{m}$ presents the link $m$ in the network, and $1 \leq m \leq n(n-$ 1).

Definition 2 Routing Model: Here, we take the $\left\{e_{i},\left(t_{s i}, t_{e i}\right)\right\}$, and $1 \leq i \leq n(n-1)$ as one link, and $t_{s i}, t_{e i}$ as the starting time and end time of the link respectively, and $t_{e i}>t_{s i}$. In opportunistic networks, there must be one link set at least, such as $\sum\left\{e_{i},\left(t_{s i}, t_{e i}\right)\right\}$, of which the links connect with ech other logically, and that the starting node and the end node are corresponding with the source node and the destination node of the data packet. Moreover, the starting time of the current link $t_{s}$ must be smaller than the end time of the next link $t_{e}$. That is, $t_{s i}<t_{e(i+1)}$.

\section{B. Problem Description}
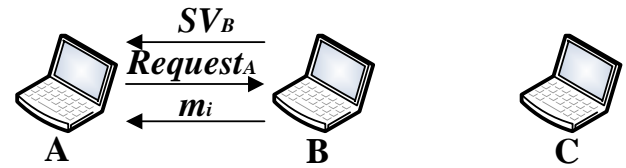

Figure 1. Data exchange for multi-nodes case

Figure 1 illustrates a possible epidemic routing scenery. In epidemic routing algorithm, when node $B$ receives a Hello packet from node A, it will send $S V_{B}$ maintained by itself to node A. Through comparison of $S V_{B}$ and $S V_{A}$ held by A itself, node A determines which packets node B holds while A does not, and derives the index, Request $t_{A}$, for these data packets. Here, Request $_{A}$ is

$$
\text { Re } \text { quest }_{A}=S V_{B}+\overline{S V_{A}}
$$

Then, node A sends Request ${ }_{A}$ to node $\mathrm{B}$. When node $\mathrm{B}$ receives the Request packet, it will send the requested data packets one by one. Here, due to the unicast communication, node $\mathrm{B}$ and node $\mathrm{C}$ will not initiate data transmission, resulting in the waste of the temporary contact time, and therefore the increment of packet delivery delay.

Moreover, the original epidemic routing pays no consideration to the priority for data packet transmission, and lacks the scheme of buffer managing for the data packets already reached the destination nodes. All these items leads to the increment of packet delivery delay, buffer occupancy, network overhead and so on.

\section{THE XNCER ALGORITHM}

In this section, we formulate the novel scheme from the aspects of algorithm operations, assurance of key parameters as well.

\section{A. Algorithm Operation}

The data exchange of XNCER algorithm is depicted as figure 2 .

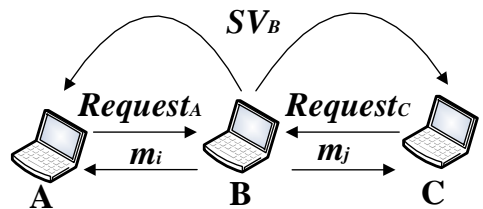

Figure 2. The data exchange of XNCER algorithm

The concrete operations for XNCER algorithm are as follows:

a) On receiving a Hello message, node B broadcasts $S V_{B}$ held by itself.

b) On getting the Request ${ }_{A}$, node $\mathrm{B}$ initiates the waiting time counter $T$, and determines the data packet set $M_{A}$ to be transmitted to node A.

c) Node $\mathrm{B}$ checks if there is any data packet in $M_{A}$ destined to node $\mathrm{A}$. If so, node B unicasts the packet to node A immediately. If the corresponding ACK is obtained, node $\mathrm{B}$ removes the data packet from its buffer at once. If the ACK does not arrive, node B must not delete the data packet. If there is no data packet destined to node A, go to the next step.

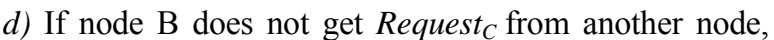
that is, node $\mathrm{C}$ during the time interval $T$, the data packets in $M_{A}$ must be delivered to node A right now. Otherwise, go to the next step.

e) Node B determines the set of data packets, that is $M_{C}$, to be transmitted to node $\mathrm{C}$. Then, similar to step $c$ ), node $\mathrm{B}$ checks if there is any data packet in $M_{C}$ destined to node $\mathrm{C}$. If so, node $\mathrm{B}$ unicasts the packet to node $\mathrm{C}$ immediately. If the corresponding $\mathrm{ACK}$ is obtained, node $\mathrm{B}$ removes the data packet from its buffer at once. If the ACK does not arrive, node $\mathrm{B}$ must not delete the data packet. If there is no data packet destined to node $\mathrm{C}$, go to the next step.

f) Node B takes one data packet only existes in $M_{A}$ and another data packet only existes in $M_{C}$ respectively, XORs them into a coded packet and broadcasts it out.

g) Node B combines the remanent data packets into the set $M_{A C}\left(M_{A C}=M_{A} \cup M_{C}\right)$, and broadcasts the corresponding data packets.

\section{B. Confirmation of the waiting time threshold $T$}

The waiting time threshold is defined as follows: When node $\mathrm{B}$ gets $S V_{A}$, the longest time that node $\mathrm{B}$ can wait before sends the corresponding data packets to node $\mathrm{A}$. In order to find the coding opportunities as more as possible and, at the meantime, guarantee the successful delivery of the data packets to be sent to node A. Here, we have the waiting time threshold $T$ as follows:

$$
T=\frac{R-d}{2 \times V_{\max }}-N \times\left(S_{F}+t_{\max }\right) \times(D+1)
$$


Here, $R$ denotes communication range, $d$ presents the distance between the pairwise nodes, and it can be measured through the mechanism of RSSI(Received Signal Strength Indicator)[12]. $V_{\max }$ Indicates the maximum rate for node mobility. $N$ is the number of data packets to be sent to node A. $S_{F}$ is the time for inter-frame. $D$ denotes the node degree, while $t_{\max }$ presents the time for transmitting the longest data frame, and it can be derived from the following equation.

$$
t_{\max }=\frac{N_{\max }}{R_{0}}+\tau_{1}+\tau_{2}
$$

Where, $R_{0}$ denotes the data rate. $\tau_{1}$ and $\tau_{2}$ are the time for transmitting Preamble field and SIGNAL field respectively. $N_{\max }$ is the maximum number of bits for each frame, and it is:

$$
\begin{aligned}
N_{\text {max }}= & \operatorname{ceil}\left(\left(N_{\text {Service }}+N_{\text {Header }}+N_{\text {Dmax }}\right.\right. \\
& \left.\left.+N_{\text {FSC }}+N_{\text {Tail }}\right) / N_{S}\right) \times N_{S}
\end{aligned}
$$

Here, ceil is the function to get the minimum integer that is not smaller than the practical number.

Hence, the waiting time threshold $T$ is ultimately confirmed according to the distance to the neighboring node and the number of data packets to be transmitted.

\section{SIMULATION AND ANALYSIS}

The OPNET14.5 network simulator is adopted to model and simulate the related algorithms. Under the same simulation conditions, the performance comparison and analysis are executed among XNCER algorithm, Direct Delivery(DD) algorithm, Epidemic Routing (ER) algorithm and Wait and Epidemic (WE) algorithm in terms of network overhead, average end-to-end delay and data packet delivery rate and so on.

\section{A. Simulation Parameters}

The key simulation parameters used in the experiment are as follows

TABLE I . KEY SIMULATION PARAMETERS

\begin{tabular}{|c|c|}
\hline Parameter & Value \\
\hline node number & 50 \\
\hline simulation area & $1500 \mathrm{~m} \times 300 \mathrm{~m}$ \\
\hline mobile model & Random waypoint \\
\hline rate range & {$[1,19](\mathrm{m} / \mathrm{s})$} \\
\hline pause time & $0 \mathrm{~s}$ \\
\hline communication range & $10 \mathrm{~m}, 25 \mathrm{~m}, 50 \mathrm{~m}, 75 \mathrm{~m}, 100 \mathrm{~m}$ \\
\hline simulation time & $2500 \mathrm{~s}$ \\
\hline maximum data rate & $54 \mathrm{Mbps}$ \\
\hline data packet size & $1 \mathrm{~KB}$ \\
\hline data send interval & $1 \mathrm{~s}$ \\
\hline send starting time & $5 \mathrm{~s}$ \\
\hline send end time & $1984 \mathrm{~s}$ \\
\hline buffer size & $2000 \mathrm{~KB}$ \\
\hline random seed & $128,130,132,134$ \\
\hline
\end{tabular}

The simulation sceneries are analogy to the literature[1]. In order to avoid the randomness of the simulation results, we have the random seed $\in\{128,130,132,134\}$. Therefore, each simulation is performed for four times, and the mean value of the simulation results is extracted as the final result.

\section{B. Result Analysis}

1) Network overhead: Network overhead means the total bits sent by all nodes, including Hello packets, SV packets, Request packets and data packets as well, during the network running time.

$$
\begin{aligned}
C_{\text {total }}= & N_{H} \times S_{H}+N_{S V} \times S_{S V} \\
& +N_{\mathrm{R}} \times S_{\mathrm{R}}+N_{D} \times S_{D}
\end{aligned}
$$

Here, $C_{\text {total }}$ denotes the total network overhead. $S_{H}$ and $N_{H}$ mean the size of Hello packet and the total number sent during the network running time respectively. Similarly, $S_{S V}$ and $N_{S V}$ are the corresponding size and number of $S V$ packet. $S_{R}$ and $N_{R}$ are the corresponding size and number of Request packet. $S_{D}$ and $N_{D}$ are the corresponding size and number of data packet.

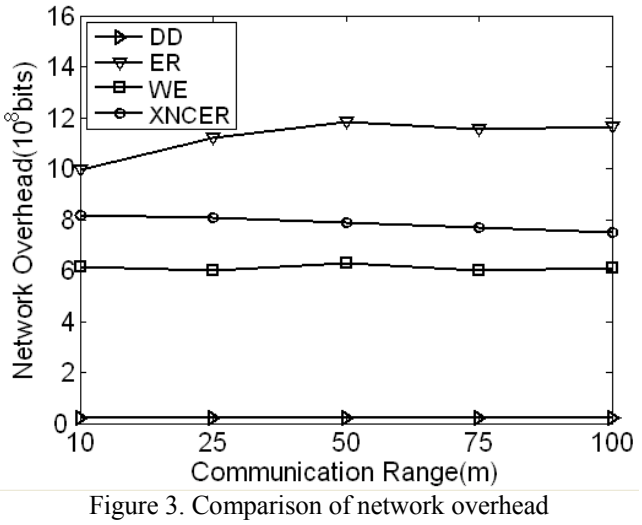

Figure 3 shows that network overhead of XNCER algorithm is remarkably lower than Epidemic Routing algorithm in each scenery. The reasons are as follows. First of all, XNCER algorithm broadcasts SV control packets(instead of unicasting), so as to reduce the control overhead and initiate the data exchange more timely. Moreover, XOR network coding is introduced to the algorithm, and thus cuts down the transmission time of data packets. Furthermore, XNCER algorithm deletes some data packets, which have already arrived at the destination nodes. Consequently, the dissemination of redundant data packets is restrained to a relatively low level. Although Direct Delivery algorithm and Wait and Epidemic algorithm cut down the number of data packets over the network significantly, neither of them pay enough attention to the use of network information resource, resulting in large transmission delay, as we will show in the next section.

2) Data packet average end-to-end delay: The end-to-end delay of a data packet includes the time the data packet is stored and carried, and the time for transmission between nodes. The data packet average end-to-end delay can be derived from the following equation.

$$
T_{\text {avg }}=\frac{\sum_{i=1} T_{i}}{N_{\text {num }}}
$$

Here, $T_{\text {avg }}$ presents the packet average end-to-end delay, $T_{i}$ indicates the delay of the ith data packet arrived at its destination node. $N_{\text {num }}$ denotes the number of data packets already reached their destination nodes. 


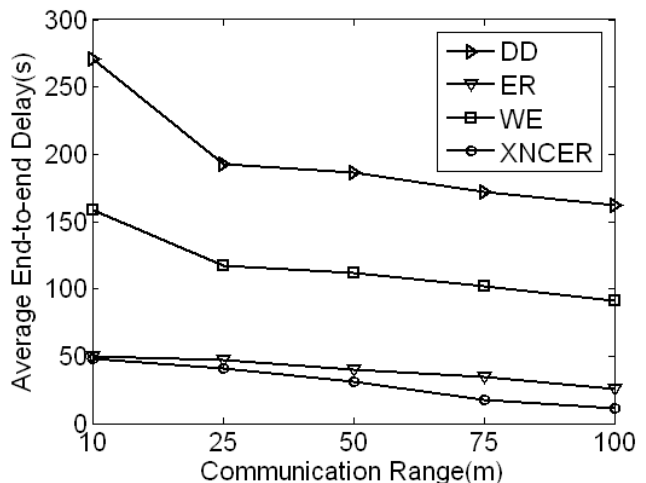

Figure 4. Comparison of average end-to-end delay

Figure 4 depicts that, average end-to-end delay of the four algorithms decrease as the increment of communication range. The expansion of node communication range induces the augmentation of node contact opportunity, fasterring the data transmission. Average end-to-end delay of XNCER algorithm is apparently lower than the others. Through broadcasting the $S V$ control packets, XNCER algorithm can initiate the data exchange immediately, make full use of short lived contact time of nodes and deliver more data packets. Moreover, nodes can use current local network topology and utilize the advantage of network coding in advancing the efficiency of data transmission. Furthermore, XNCER algorithm sends the data packets destined to the one hop neighboring nodes swiftly, and delete some of them in node buffers according to the corresponding ACKs. All these schemes cut down the delivery delay.

3) Data packet delivery rate: The data packet delivery rate is the ratio of the number of data packets successfully reached their destination nodes to the number of data packets the source nodes generated and sent out. The value is given as follows.

$$
D_{\text {rate }}=\frac{\sum_{i=0} D_{i}}{\sum_{i=0} S_{i}}
$$

Here, $D_{\text {rate }}$ means the data packet delivery rate, $D_{i}$ denotes the number of data packets arrived at the destination node i, $S_{i}$ indicates the number of data packets the source node $i$ generated and sent out. The data packet delivery rate of all the four algorithms are showed in the following table.

TABLE II. COMPARISON Of DATA PACKET DELIVERY RATE

\begin{tabular}{|c|c|c|c|c|}
\hline CR $(\mathrm{m})$ & DD & ER & WE & XNCER \\
\hline 10 & $97.07 \%$ & $100 \%$ & $100 \%$ & $100 \%$ \\
\hline 25 & $99.65 \%$ & $100 \%$ & $100 \%$ & $100 \%$ \\
\hline 50 & $99.24 \%$ & $100 \%$ & $100 \%$ & $100 \%$ \\
\hline 75 & $99.19 \%$ & $100 \%$ & $100 \%$ & $100 \%$ \\
\hline 100 & $99.44 \%$ & $100 \%$ & $100 \%$ & $100 \%$ \\
\hline
\end{tabular}

Here, CR means the Communication Range. From Table II, we can see that Epidemic Routing algorithm, Wait and Epidemic routing algorithm as well as XNCER algorithm get a high data packet delivery rate up to $100 \%$, and have a better robustness. This is because that all these three algorithms are flooding-based. However, due to the randomness for node mobile, single-copy routing algorithms, such as Direct Delivery algorithm, can seldom obtain a completely delivery.

\section{CONCLUSIONS}

Through broadcasting $S V$ control packets, utilizing the XOR network coding, sending data packets destined to the next hop neighboring nodes immediately, and deleting some of them according to the received ACKs running in the MAC layer, XNCER algorithm can take full advantage of current node local network topology, perform neighboring discovery timely and initiate data exchange immediately as well as enhance the efficiency of data transmission, so as to cut down the network overhead, shorten the data packet average end-to-end delay and improve the data packet delivery rate.

\section{ACKNOWLEDGMENT}

This work was supported in part by National Natural Science Foundation of China under Grant No. 60972068, the Scientific Research Starting Foundation for Returned Overseas Chinese Scholars, Ministry of Education of China under Grant No. 2010-1561, the Science Research Project of Education Committee of Chongqing under Grant No. KJ120510, and the project of Emergency Communication Laboratory of Chongqing under Grant No. 201201.

\section{REFERENCES}

[1] A. Vahdat, D. Becker, Epidemic Routing for Partially Connected Ad Hoc Networks. Technical Report CS-200006, Duke University, 2000.

[2] L. Pelusi, A. Passarella, and M. Conti, "Opportunistic networking: data forwarding in disconnected mobile ad hoc networks," IEEE Communications Magazine, vol. 44, pp. 134-141, 2006.

[3] R. Ahlswede, N. Cai, and S.Y.R. Li, "Network information flow," IEEE Trans Inform Theory, vol. 46, pp. 1204-1216, 2000.

[4] H. Wen, F.Y. Ren, and J. Liu, "A storage-friendly routing scheme in intermittently connected mobile network," IEEE Transactions on Vehicular Technology, vol. 60, pp. 1138-1149, 2011.

[5] J. Z. Sun, Y.Y. Xiao, and H.Q. Chen, Wait and Epidemic Routing Algorithm. China Patent, CN201110130398.7, 2011.

[6] M. Grossglauser, D.N.C. Tse, "Mobility increases the capacity of ad hoc wireless networks," IEEE/ACM Trans. on Networking, Vol. 10, pp. 477-486, 2002.

[7] S. Katti, H. Rahul, and W. Hu, "Xors in the air: practical wireless network coding," IEEE/ACM Transactions on Networking, Vol. 16, pp. 497-510, 2008.

[8] X.M. Wang, J.P. Wang, Y.L. Xu, "Data dissemination in wireless sensor networks with network coding," EURASIP Journal on Wireless Communication and Networking, pp. 1-14, 2010.

[9] Y.F. Lin, B.C. Li, and B. Liang, "Stochastic analysis of network coding in epidemic routing," IEEE Journal on Selected Areas in Communications, vol. 26, pp. 794-808, 2008.

[10] S.K. Yoon, Z.J. Haas, "Application of linear network coding in delay tolerant networks," The Second International Conference on Ubiquitous and Future Networks (ICUFN 2010), IEEE Press, 2010, pp. 338-343.

[11] B.K. Zhao, Z.M. Song, and J.S. Su, "NTC: Towards efficient network coding in delay tolerant networks," 2011 Fifth International Conference on Innovative Mobile and Internet Services in Ubiquitous Computing, IEEE Press, 2011, pp. 57-64.

[12] F. Mourad, H. Snoussi, and C. Richard, "Interval-Based Localization using RSSI Comparison in MANETs," IEEE Transactions on Aerospace and Electronic System, vol. 47, pp. 2897-2910, 2011. 